\title{
Dosage du quartz dans la poussière de molasse par la technique infrarouge
}

\author{
Marie-Antoinette Bianco*, Corinne Burla et Jean-Claude Landry
}

Abstract. An IR technique for the determination of quartz in sandstone dusts is described. This technique is particle-size sensitive; this is well-illustrated with the analyses of dusts collected on different stages of an impactor. Pellets were prepared to obtain precise quantitative determination of quartz either by the conventional dilution method or after wet grinding with $\mathrm{EtOH}$. The alcoholic treatment proved far to be superior to the dry homogenization. Finally, different ways of quantification of quartz IR spectroscopy are compared: absorbance at $798 \mathrm{~cm}^{-1}$, integrated area measurement which includes both bands, 'QUANT' software of Perkin-Elmer. The 'QUANT' software proved to be the easier and the most reliable means of quantitation. A comparison between IR spectrometry and X-ray diffraction is equally reported. This method has been used in a survey of the quartz dust concentration to which stoneworkers are exposed; these results will be given in another publication.

\section{Introduction}

Cette étude a pour but la mise au point d'une méthode de quantification du quartz dans des poussières de molasse par spectrométrie infrarouge à transformée de $\mathrm{Fou}$ rier (FT-IR) et l'utilisation de cette méthode dans l'évaluation de l'exposition des tailleurs de pierre et plus spécialement des rénovateurs de façades aux poussières de quartz. Nous donnons ci-après une description de la méthode et des problèmes rencontrés lors de sa mise au point.

\section{Matériel et méthodes}

\subsection{Prélèvements de poussière dans l'air}

Pour les prélèvements de longue durée, 8 h et plus, des pompes Dupont $\mathrm{P} 4000$, réglées à un débit de $1,9 \mathrm{l} / \mathrm{min}$, ont été utilisées. La séparation des poussières fines a été effectuée à l'aide d'un cyclone SKC No 225-01-02 couplé à un filtre membrane (ester de cellulose Millipore HAWP037) de $37 \mathrm{~mm}$ de diamètre et de $0,45 \mu \mathrm{m}$ de porosité.
Pour les prélèvements de courte durée, de 10 min à $1 \mathrm{~h}$, un impacteur Andersen, type $1 \mathrm{AFCM}$ à 7 étages, a été utilisé.

\subsection{Analyses}

La pesée des échantillons a été faite sur une balance de précision Mettler AT261. Le dosage du quartz a été effectué par spectrophotométrie infrarouge sur un appareil FT-IR Perkin-Elmer No. 1720X. Les échantillons ont été dispersés dans des pastilles de $\mathrm{KBr}$. La quantification s'est effectuée en prenant soit l'absorbance d'un des doublets du quartz à $798 \mathrm{~cm}^{-1}$, soit la surface comprise entre 820 et $770 \mathrm{~cm}^{-1}$ comprenant les deux doublets. Un logiciel de quantification développé par Perkin-Elmer sous le nom de 'QUANT' a également été utilisé. 'QUANT' est un modèle numérique de reconstitution de spec- tre expérimental à partir de spectres de références permettant une évaluation quantitative.

Les pastilles de $\mathrm{KBr}$ ont été préparées de la manière suivante: on broye l'échantillon dans un broyeur à billes en agate. Une fraction de l'échantillon ( $1 \mathrm{mg}$ ) est pesée puis broyée dans un mortier en présence de EtOH. On ajoute alors la quantité adéquate de bromure de potassium, ( 300 $\mathrm{mg}$ ) de $\mathrm{EtOH}$ et on continue le broyage. Les standards ont été préparés par dilutions successives à partir d'une soln. mère dans le $\mathrm{KBr}$ préparée comme décrit ci-dessus.

Une comparaison des résultats obtenus par notre technique et par la technique de diffraction de rayons $\mathrm{X}$ a été effectuée sur un diffractomètre Philips 1361 par la méthode des ajouts.

\section{Résultats}

\subsection{Effet du broyage en présence de $\mathrm{EtOH}$}

Cette méthode de préparation est celle décrite par Hlavay et Inczédy [1]. Si on compare le même échantillon, broyé dans un mortier en agate, mélangé au $\mathrm{KBr}$ à sec ou en présence de $\mathrm{EtOH}$, on constate les différences définies ci-après:

- bien meilleure résolution du doublet du quartz, le pic à $798 \mathrm{~cm}^{-1}$ est plus grand que celui à $779 \mathrm{~cm}^{-1}$;

- grande amélioration de la sensibilité: pour une concentration de quartz de 1 mg dans la pastille, l'absorbance à 798 $\mathrm{cm}^{-1}$ est de $0,79+0,02$ alors que sans EtOH l'absorbance pour la même concentration n'est plus que de $0,37+$ 0,01 .

\subsection{Effet de la granulométrie sur la concentration apparente de quartz}

Une façon élégante de mettre en évidence ce phénomène est l'analyse de la poussière retenue sur les différents étages de l'impacteur, lors des prélèvements de poussière dans l'air. Chaque étage correspond en effet à une granulométrie précise. Dans le tab. 1 nous donnons les résultats obtenus pour la teneur en quartz dans des prélèvements sur les différents étages de

Tab. 1. Effet de la granulométrie sur la concentration apparente de quartz

\begin{tabular}{lll}
$\begin{array}{l}\text { Taille des } \\
\text { particules }[\mu \mathrm{m}]\end{array}$ & $\begin{array}{l}\text { Concentration de quartz } \\
\text { sans broyage }[\%]\end{array}$ & $\begin{array}{l}\text { Concentration de quartz } \\
\text { après broyage }[\%]\end{array}$ \\
\hline$>10$ & 13,6 & 49,5 \\
de 9 à 10 & 17,3 & 50,3 \\
de 5,8 à 924,4 & 24,4 & 51,1 \\
de 4,7 à 5,8 & 30,2 & 48,2 \\
de 3,3 à 4,7 & 37,7 & 48,5 \\
de 2,1 à 3,3 & 46,2 & 48,0
\end{tabular}


Tab. 2. Comparaison de trois méthodes différentes de quantification

\begin{tabular}{|c|c|c|c|}
\hline & \multicolumn{2}{|c|}{ Méthodes de quantification } & \multirow[b]{2}{*}{$\begin{array}{l}\text { 'QUANT' } \\
820 \text { à } 770 \mathrm{~cm}^{-1}\end{array}$} \\
\hline & $\begin{array}{l}\text { Absorbance à } \\
798 \mathrm{~cm}^{-1}\end{array}$ & $\begin{array}{l}\text { Surface entre } \\
820 \text { et } 770 \mathrm{~cm}^{-1}\end{array}$ & \\
\hline $\begin{array}{l}\text { Echantillon } \\
\text { non broyé } \\
\text { dans EtOH }\end{array}$ & 13,7 & 11,1 & 10,6 \\
\hline $\begin{array}{l}\text { Echantillon } \\
\text { broyé } \\
\text { dans EtOH }\end{array}$ & 38,3 & 34,5 & 33,3 \\
\hline
\end{tabular}

Remarque: Dans cet échantillon dosé par diffraction de rayons X le pourcentage mesuré était de $32 \%$.
Le logiciel 'QUANT' a finalement été adopté du fait de sa bonne reproductibilité, de sa facilité d'utilisation et de sa bonne concordance avec les résultats obtenus par rayons $\mathrm{X}$.

\subsection{Application de la méthode au dosage du quartz dans l'air}

La toxicité du quartz sur l'organisme est liée à la granulométrie. La séparation des poussières fines des poussières grossières est donc impérative lors du prélèvement d'air. Pour cela, le couplage d'un cyclone à un filtre membrane est utilisé. Seules les poussières retenues sur le filtre sont prises en compte pour l'analyse. Pour l'impacteur avec et sans broyage dans le mortier en agate. On constate que plus la taille des particules est grande, plus la concentration apparente de quartz diminue (tab. l). La granulométrie des particules affecte l'absorbance et la résolution du doublet du quartz (fig.). Le phénomène est dû à la diffraction et à la réflection du rayon incident par les particules de taille $\geq$ à $2,5 \mu \mathrm{m}$. Il a d'ailleurs déjà été mis en évidence également par d'autres auteurs [1-4].

\subsection{Méthodes de quantification du quartz}

La quantification en IR pour le quartz se fait généralement en prenant l'absorbance de la bande à $798 \mathrm{~cm}^{-1}$. Mais cette bande est très influencée par les différences de granulométrie. Certains auteurs ont donc suggéré de considérer non plus cette bande mais de calculer la surface comprenant les deux bandes d'absorption du doublet, soit la surface comprise entre 820 et $770 \mathrm{~cm}^{-1}$ [5] . Ces deux procédures ainsi que la méthode 'QUANT' ont été comparées. Le même échantillon avec et sans broyage a été dosé au moyen de chacune des trois méthodes. Il s'agissait d'un échantillon de molasse bleue pour lequel nous avions également le résultat obtenu par rayons $\mathrm{X}$.

Les résultats obtenus sont donnés dans le $t a b .2$; ils sont exprimés en pourcent. Selon la théorie proposée par Cares et al. [5], la quantification utilisant le calcul de la surface devrait donner un pourcentage de quartz comparable dans le même échantillon avec ou sans broyage. On constate que ce n'est pas le cas. En effet, sans broyage, le pourcentage de quartz reste bien inférieur à la réalité quelle que soit la méthode de quantification utilisée. La préparation de l'échantillon a une importance primordiale pour l'obtention d'un résultat correct. Les trois méthodes peuvent être utilisées indifféremment.

\section{Echantillons non broyés}
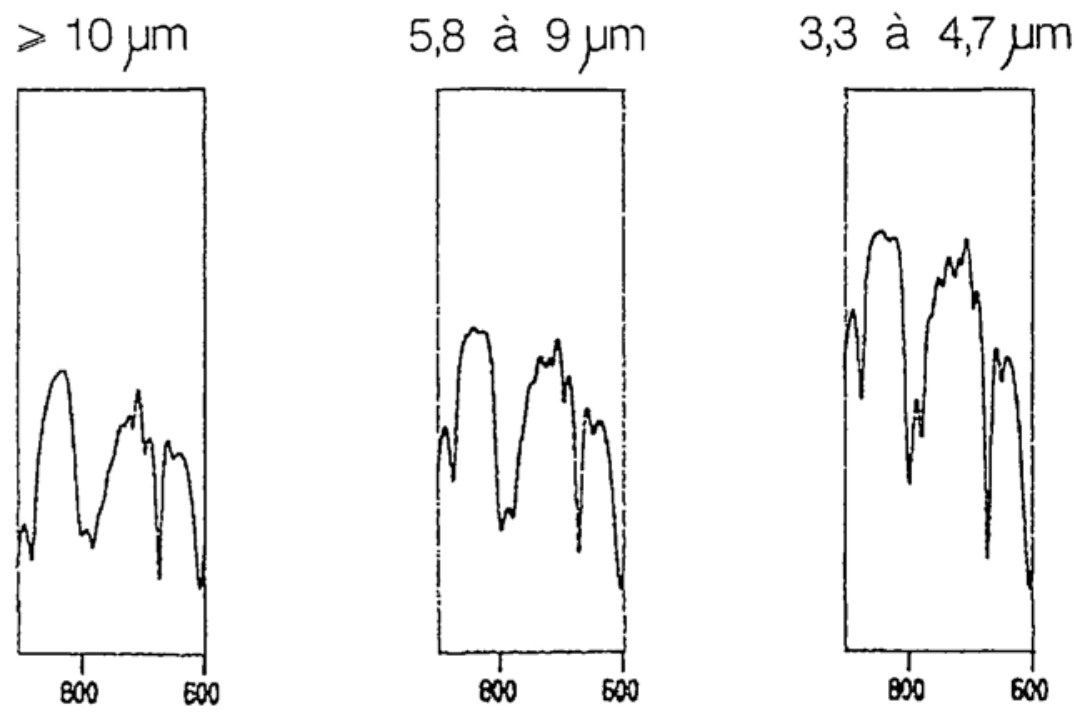

\section{Echantillons broyés}
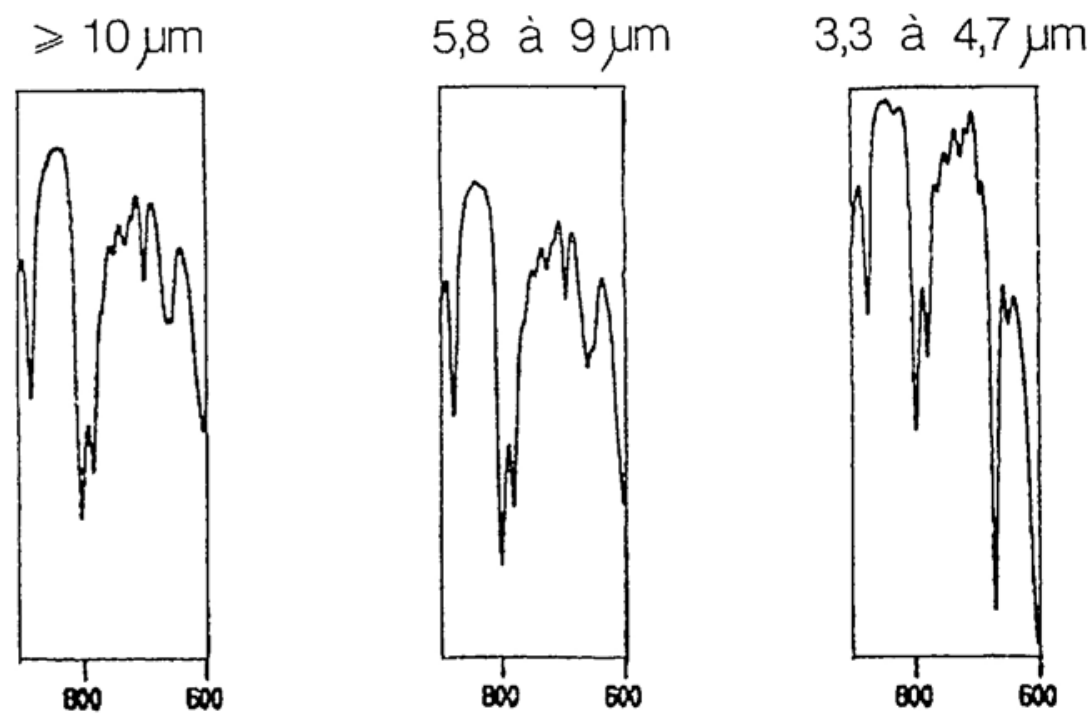
ce type de poussières, le broyage dans le broyeur à billes en agate n'est pas nécessaire; un broyage dans le mortier en présence de $\mathrm{EtOH}$ lors de la préparation de la pastille de $\mathrm{KBr}$ est suffisant pour obtenir une granulométrie comparable au standard.

\subsection{Comparaison avec la diffraction de rayons $X$}

La concordance des résultats obtenus pour le dosage du quartz dans deux échantillons de poussière de molasse par FT-IR avec notre méthode de préparation et par diffraction de rayons $\mathrm{X}$ est bonne ( $t a b .3)$. Lors de cette comparaison, la quantification du quartz a été faite avec le logiciel 'QUANT'.

\section{Conclusions}

La méthode de dosage du quartz par FT-IR décrite ici permet d'obtenir des résultats comparables à ceux obtenus par diffraction de rayons X. Elle est adaptée au dosage du quartz dans la poussière de

Tab. 3. Comparaison des résultats obtenus par FT-IR et par diffraction de rayons $X$

\begin{tabular}{lll} 
Echantillon & $\begin{array}{l}\text { FT-IR } \\
\text { concentration } \\
{[\%]}\end{array}$ & $\begin{array}{l}\text { Diffraction } \\
\text { rayon } X \\
\text { concentration }[\%]\end{array}$ \\
\hline 1 & 26,5 & 25 \\
2 & 33,3 & 32
\end{tabular}

molasse. La standardisation de la méthode de préparation des pastilles de $\mathrm{KBr}$ est primordiale pour une bonne reproductibilité. L'étape de broyage humide dans EtOH proposée par Hlavay et Inczedy [1] pour d'autres substances minérales s'est révélée particulièrement bien adaptée au quartz.

Cette méthode a été appliquée avec succès pour le dosage de l'exposition au quartz des tailleurs de pierre lors de travaux de ravalement de façades.

Nous remercions le Prof. Michel Delaloye et Madame Michèle Senn, sa collaboratrice, au Département de Minéralogie de la Faculté des Sciences de l'Université de Genève, pour avoir effec- tué le dosage par diffraction de rayons $\mathrm{X}$ et Madame N. Perruchoud pour son assistance technique.

Reçu le 21 janvier 1992

[1] J. Hlavay, J. Inczédy, Spectrochim. Acta, Part A 1985, 4l, 783.

[2] G. Duyckerts, Analyst 1959, 84, 201.

[3] J. Hlavay, L. Antal, I. Vassanyi, J. Karpati, Acta Phys. Hung. 1988, 63, 109.

[4] P. L. Anderson, Am. Indust. Hyg. Assoc. J. $1975,36,767$.

[5] J. W. Cares, A. S. Goldin, J. J. Lynch, W. A. Burgess, Am. Indust. Hyg. Assoc. J. 1973, 34, 298. 
Schweizerische Chemische Gesellschaft Société Suisse de Chimie

\section{Einladung zur (letzten) Mitgliederversammlung der} Schweizerischen Chemischen Gesellschaft

Dienstag, 21. April 1992, 16.30 h

Hörsaal Rouiller

Universität Dufour

24, rue du Général Dufour

$\mathrm{CH}-1211$ Genève 4

\section{Traktanden}

1. Protokoll der Herbstversammlung vom 18. Oktober 1991 in Bern Procès-verbal de l'assemblée d'automne du 18 octobre 1991 à Berne

2. Bericht des Vorstandes über das Jahr 1991 Rapport du Comité pour l'année 1991

3. Kassabericht und Abnahme der Jahresrechnung 1991 Rapport du trésorier et approbation des comptes de l'exercice 1991

4. Helvetica Chimica Acta

5. Gründung der 'Neuen Schweizerischen Chemischen Gesellschaft' Fondation de la 'Nouvelle Société Suisse de Chimie'

6. Auflösung der 'Schweizerischen Chemischen Gesellschaft' (2. Lesung gemäss Art. 29 der Statuten) Dissolution de la 'Société Suisse de Chimie' (2ème votation selon Art. 29 des Statuts)

7. Varia/divers

Anschliessend findet ab $17.30 \mathrm{~h}$ ein Empfang durch den Conseil d'Etat du Canton et République de Genève statt.

Der Präsident:

Prof. W. von Philipsborn

Der Sekretär:

Dr. E. Zass

\section{Protokoll}

der Herbstversammlung der Schweizerischen Chemischen Gesellschaft vom 18. Oktober 1991 in Bern

\section{A. Geschäftlicher Teil}

Der Präsident eröffnete die Sitzung um 8.30 Uhr.

1. Das Protokoll der Frühjahrsversammlung vom 15. März 1991 in Basel wird durch Akklamation genehmigt.

2. Die Frühjahrsversammlung 1992 wird in erweitertem Rahmen als internationales Symposium 'Organic Chemistry: Its Language and Its State of the Art' aus Anlass des 100-Jahr-Jubiläums der 'Geneva Conference' vom 22.-24. April in Genf stattfinden. $\mathrm{Zu}$ diesem Anlass wird eine Sonderbriefmarke der PTT herausgegeben. Das Organisationskomitee unter Leitung von M.V. Kisakürek konnte folgende Vortragende gewinnen: M.C. Caserio (Univ. of California, Irvine, U.S.A.), J.D. Dunitz

(ETH-Zürich), E. Godly (Lab. of Government Chemists, London, U.K.), P.A. Grieco (Indiana Univ., Bloomington, U.S.A.), L.S. Hegedus (Colorado State Univ., Fort Collins, U.S.A.), M.V. Kisakürek (Helvetica Chimica Acta, Basel), J.-M. Lehn (Univ. Louis Pasteur, Strasbourg, France), K.L. Loening (Topterm, Columbus, U.S.A.), K. Müller (F. Hoffmann-La Roche AG, Basel), W. Oppolzer (Univ. de Genève, Genève), L.A. Paquette (Ohio State Univ., Columbus, U.S.A.), V. Prelog (ETH-Zürich), J.H. Stocker (Univ. of New Orleans, New Orleans, U.S.A.), J.G. Traynham (Louisiana State Univ., Baton Rouge, U.S.A.). Ein erstes Zirkular zu diesem Symposium wurde bereits versandt, das zweite ist in Vorbereitung.

3. Die Texte zur Statutenänderung (Art. // und 22) werden vorgelegt - der deutsche Text wurde mit der Einladung den Mitgliedern zugestellt - und in zweiter Lesung einstimmig genehmigt.

4. Der Präsident informiert über den Stand der Vorbereitungen zur Gründung der 'Neuen Schweizerischen Chemischen Gesellschaft' (NSCG), deren Statuten vom Vorstand der SCG in der Sitzung vom 17.10.91 verabschiedet wurden. Die NSCG soll parallel zu den beiden anderen Gesellschaften existieren, bis diese im Frühjahr 1992 rückwirkend auf den 1.1.1992 aufgelöst werden. Dies ist für die Mitgliederversammlung am 21.4.92 in Genf vorgesehen; rechtzeitig vor dieser Sitzung werden alle Mitglieder schriftlich über die dort zu fassenden Beschlüsse informiert werden und die Statuten der NSCG erhalten. Nach Verlesung von Art. 29 und 27 der Statuten, betreffend das Prozedere zur Auflösung der SCG, wird der Antrag des Vorstandes zur Diskussion gestellt: 'Ermächtigen Sie den Vorstand, eine 'Neue Schweizerische Chemische Gesellschaft' zu gründen, sobald sich ein engerer Vorstand für die neue Gesellschaft konstituiert hat, dessen Mitglieder später durch Wahl zu bestätigen sind? Dieser neuen Gesellschaft gehören die Mitglieder der SCG und des SChV nach der erfolgten Genehmigung der neuen Statuten und der dann zu beschliessen Auflösung der beiden alten Gesellschaften an.' Dieser Antrag ist als erste Lesung einer Auflösung der SCG mit endgültiger Abstimmung am 21.4 .92 zu verstehen. Darauf wird der Antrag einstimmig angenommen.

5. Varia: Ein Mitglied verleiht seiner Freude über die Verleihung des Nobelpreises für Chemie an Prof. Richard Ernst, ETH-Zürich Ausdruck; die Versammlung reagiert mit spontanem Beifall.

Schluss der Sitzung: 8.55 Uhr

\section{B. Wissenschaftlicher Teil}

Das dieses Jahr besonders umfangreiche und gut besuchte Programm enthielt den Vortrag des Werner-Preisträgers 1991, Dr. B. Ernst (CibaGeigy $A G$, Basel) 'Katalytische Glykosilierungsreaktionen am Beispiel eines Signalstoffes zur Insektenbekämpfung' und 42 Kurzmitteilungen in Organischer Chemie, 8 Vorträge in der Medizinischen Chemie sowie Kurzmitteilungen der Sektionen Radiochemie (25), Physikalische Chemie (9) und Computerunterstützte Chemie (10). Die Anorganische Chemie veranstaltete ihr traditionelles Minisymposium zum Thema 'Coordination Compounds in Medicine' mit Beiträgen von Th.A. Kaden (Univ. Basel), P.J. Sadler (Univ. of London/England) 'The design of metal complexes as drugs and diagnostic agents', J.F. Desreux (Univ. de Liège/Belgium) 'Paramagnetic contrast agents for NMR imaging', $P$. Bläuenstein et al. (Paul Scherrer Institut, Villigen) 'Complexes of Tc (and Re) in relation to nuclear medicine' und $H$. Mäcke (Kantonsspital Basel) 'Approaches to monoclonal antibody labeling by use of metallic radionuclides' und eine Postersession mit 67 Postern. Die an dieser Versammlung neugegründete Sektion 'Analytische Chemie' organisierte ein Seminar mit 6 Vorträgen und 42 Postern.

Der Präsident:

Prof. W. von Philipsborn
Der Sekretär:

Dr. E. Zass 


\section{Schweizerischer Chemiker-Verband} Schweizerische Chemische Gesellschaft

6th International Seminar on

\section{Modern Synthetic Methods}

Interlaken, Switzerland, May 4/5, 1992

Chairman: Prof. Rolf Scheffold, University of Berne

Monday, May 4, 1992

Lectures: Auditorium Ist floor, Secondary School, Alpenstrasse 5, Interlaken

09.40-09.45 Opening by the President of the Swiss Association of Chemists

Dr. W. Graf, Lonza AG

Carbanion Chemistry

09.45-10.45 Prof. C.H. Heathcock

Modern Enolate Chemistry I:

Historical Roots, Regio- and Stereoselective Enolate Formation

10.45-11.15 Coffee break

11.15-12.15 Prof. C.H. Heathcock

Modern Enolate Chemistry II:

The Effect of Enolate Configuration on Four Important Reactions: The Aldol Reaction, the Michael Reaction, the Enolate Claisen Rearrangement, and Enolate Alkylation

12.20-13.20 Prof. E. Winterfeldt Acetylenes in Synthesis I:

Thermal Reactions, Organometallic Complexes

13.20-14.15 Business Lunch

14.15-15.15 Prof. E. Winterfeldt

Acetylenes in Synthesis II:

Ionic Additions

15.20-16.20 Prof. M. Schlosser

Superbase Chemistry I:

First-Order Principles and Neighbouring Group Effects

16.20-16.40 Coffee break

16.40-17.40 Prof. M. Schlosser

Superbase Chemistry II:

Alcoholate-Activated, Mixed Metal Reagents

19.30

Dinner

Tuesday, May 5, 1992

Lectures: Auditorium 1st floor, Secondary School, Alpenstrasse 5, Interlaken

Carbohydrate Chemistry

09.00-10.00 Prof. F.W. Lichtenthaler

Building Blocks from Sugars

10.00-10.30 Coffee break

10.30-11.30 Prof. F.W. Lichtenthaler

Sugar-Derived Building Blocks in Natural-Product Synthesis

11.40-12.40 Prof. A. Dondoni

Carbon Chain Extension:

Carbohydrate Synthesis via Thiazoles

12.40-13.30 Business Lunch
13.30-14.30 Prof. C.A.A. van Boeckel

Protective-Group Strategies in the Synthesis of Functionalized Carbohydrates, Part I

14.40-15.40 Prof. C.A.A. van Boeckel

Protective-Group Strategies in the Synthesis of Functiona-

lized Carbohydrates, Part II

15.40 Closing of the Seminar

Social events

Sunday, May 3, 1992

Welcome Cocktail

Hotel Victoria Jungfrau (Les Colonnades)

7 p.m.-8 p.m.

Participation is free of charge

Monday, May 4, 1992

Dinner on Board

Dinner on board of a ship cruising the lake of Thun.

Departure at Station Interlaken West at 7.30 p.m.

Please book for the 'Dinner on Board' together with the registration.

Registration

Secretary's Office for SAC-Symposia

Institute of Organic Chemistry, University of Berne

Freiestrasse 3, CH-3012 Berne, Switzerland

Tel. 0316543 11, Fax 031654499

Participation fee for May 4 and 5, 1992

SFr. 400.-

Reduced participation fee for members of the

Association of Swiss Chemists (SChV) and Swiss

Chemical Society (SCG)

SFr. 350.-

Participation fee for students, graduate and

post-doctoral students

SFr. 50.-

One-day participation fee for

attendance on May 4 or 5, 1992 only

For students, graduate and post-doctoral students

Sfr. 250 -

SFr. 40 .-

Included in the participation fees are:

business lunches, coffees during the breaks, file, and the book 'Modern

Synthetic Methods', Vol. 6, 1992 (ca. 450 pages).

Dinner on board

SFr. 90.-

The ticket for the dinner and boat trip is to be paid together with the registration fee.

Payment should be made to the following account:

235 855.675.01 H - Schweiz. Chemiker-Verband, Symposia

Union Bank of Switzerland, Berne

(Postcheque-No. of the Bank: 30-35-9, Berne)

or by cheque payable to the Schweizerischer Chemiker-Verband, Symposia.

Accommodation

Hotel reservations can be made with the Tourist Office Interlaken, Höhe-

weg $37, \mathrm{CH}-3800$ Interlaken, Switzerland

Tel. 036222154 or 036222121

Fax 036225221

Telex 923111

Convention Office

May 3, 1992 Hotel Victoria Jungfrau, 18.00-20.00 Uhr

May 4/5, 1992 Entrance Hall, Secondary School,

Alpenstrasse 5, Interlaken

Tel. 036224826 . 


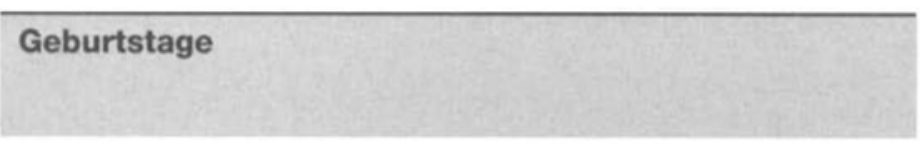

Max Rutishauser: 80. Geburtstag

Am 25. April 1992 kann Herr Dr. Max Rutishauser seinen 80. Geburtstag feiern. Anlässlich der GV vom 19.2.82 wurde er "in dankbarer Würdigung seines zielbewussten und richtungsweisenden Einsatzes als Präsident des Verbandes in den Jahren 1974 bis 1982' vom Schwei-

zerischen Chemiker-Verband zum Ehrenpräsidenten ernannt. Ein ausführliches Portrait des Jubilars erschien in Chimia 1982, 36, 176, anlässlich seines 70. Geburtstages. Der Vorstand des SChV sowie die Redaktion der Chimia entbieten die besten Glückwünsche.

\section{Akademische Ehrungen}

Prof. Dr. Albert Eschenmoser, Professor der ETH-Zürich für allgemeine organische Chemie, wurde kürzlich die Ehrendoktorwürde der Université Louis Pasteur in Strasbourg verliehen.

Prof. Dr. Dieter Seebach, Professor der ETH-Zürich für Chemie, wurde von der American Chemical Society der 'Award for Creative Work in

Synthetic Organic Chemistry' verliehen.

Prof. Dr. Wilhelm Simon, Professor derETH-Zürich für analytischeChemie, wurde die 'Talanta Medal' (Great Britain) verliehen. Unter den 13 Trägern der Talanta Medaille befinden sich auch die ehemaligen ETH-Professoren G. Schwarzenbach und $J$. Ruzicka.
7. May 1992

Prof. J. Rebek, Jr.

Massachusetts Institute of Technology, Cambridge,

USA

'Recognition and Replication in Model Systems'

\section{Chemische Gesellschaft Zürich}

Mittwoch 17.15 Uhr

Hörsaal 15-G-19 der Universität Zürich-Irchel

Winterthurerstrasse 190, 8057 Zürich

29. April 1992

Prof. Dr. H.-J. Güntherodt

Institut für Physik, Universität Basel, Basel

'Rastertunnelmikroskopie und verwandte Techniken - Abbilden, Spektroskopie, Modifikation und Manipulation auf atomarem und molekularem Niveau'

6. Mai 1992

Prof. Dr. H. Kroto

The School of Chemistry and Molecular Science, University of Sussex, Brighton, UK

' $\mathrm{C}_{60}$, Buckminsterfullerene, The Celestial Sphere that Fell to Earth'

13. Mai 1992

Prof. Dr. A. Heller

\section{Vorträge}

\section{Société Vaudoise des Sciences Naturelles}

Mercredi à $17.15 \mathrm{~h}$

Auditoire $\mathrm{C}$, Collège Propédeutique

Université de Lausanne, Dorigny

22 avril 1992

Prof. Dr. J.A. Robinson

Université de Zürich

'Probing the Relationships between Peptide Sequence, Conformation and Function at Receptors in the Immune System'

29 avril 1992

\section{Prof Dr. E. Lindner}

Université de Tübingen

"Catalytic and Stoichiometric Syntheses with Metal-Containing Heterocycles as Reactive Intermediates'
College of Engineering, The University of Texas, Austin, USA

'Molecular Wiring Networks for Electrical Connection of Redox Centers of Enzymes to Electrodes'

20. Mai 1992

Prof. Dr. S.E. Friberg

Department of Chemistry, Clarkson University, Potsdam, New York, USA

'The Structural Organization and Function of the Stratum Corneum Lipids - A Colloid Chemistry Problem'

\section{Laboratorium für Organische Chemie der ETH-Zürich}

Organisch-chemische Kolloquien

Montag, $16.30 \mathrm{Uhr}$

Hörsaal CHN A 31

27. April 1992

fällt aus wegen Sechseläuten

4. Mai 1992

\section{Prof. Dr. G. Folkers}

ETH, Departement Pharmazie, Zürich

'Active Site Directed Modelling of Viral Kinase Nucleoside Interaction Complexes'

11. Mai 1992

fällt aus wegen Abteilungskonferenz

18. Mai 1992

Prof. Dr. D.S. Kemp

Massachusetts Institute of Technology, Department of Chemistry, Cambridge, MA/USA

'Conformational Templates as Nucleation Sites and Reporter Functions for Formation of Polypeptide Helices and Sheets in Solution'

Prof. Dr. K. Müllen

Max-Planck-Institut für Polymerforschung, Mainz/ BRD

'Konjugierte Oligomere - je länger, je lieber?'

\section{Basler Chemische Gesellschaft}

Institut für Organische Chemie

Kleiner Hörsaal

$16.45 \mathrm{Uhr}$ 


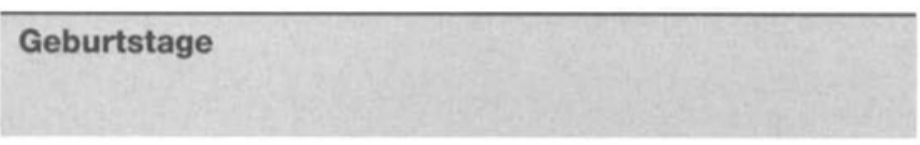

Max Rutishauser: 80. Geburtstag

Am 25. April 1992 kann Herr Dr. Max Rutishauser seinen 80. Geburtstag feiern. Anlässlich der GV vom 19.2.82 wurde er "in dankbarer Würdigung seines zielbewussten und richtungsweisenden Einsatzes als Präsident des Verbandes in den Jahren 1974 bis 1982' vom Schwei-

zerischen Chemiker-Verband zum Ehrenpräsidenten ernannt. Ein ausführliches Portrait des Jubilars erschien in Chimia 1982, 36, 176, anlässlich seines 70. Geburtstages. Der Vorstand des SChV sowie die Redaktion der Chimia entbieten die besten Glückwünsche.

\section{Akademische Ehrungen}

Prof. Dr. Albert Eschenmoser, Professor der ETH-Zürich für allgemeine organische Chemie, wurde kürzlich die Ehrendoktorwürde der Université Louis Pasteur in Strasbourg verliehen.

Prof. Dr. Dieter Seebach, Professor der ETH-Zürich für Chemie, wurde von der American Chemical Society der 'Award for Creative Work in

Synthetic Organic Chemistry' verliehen.

Prof. Dr. Wilhelm Simon, Professor derETH-Zürich für analytischeChemie, wurde die 'Talanta Medal' (Great Britain) verliehen. Unter den 13 Trägern der Talanta Medaille befinden sich auch die ehemaligen ETH-Professoren G. Schwarzenbach und $J$. Ruzicka.
7. May 1992

Prof. J. Rebek, Jr.

Massachusetts Institute of Technology, Cambridge,

USA

'Recognition and Replication in Model Systems'

\section{Chemische Gesellschaft Zürich}

Mittwoch 17.15 Uhr

Hörsaal 15-G-19 der Universität Zürich-Irchel

Winterthurerstrasse 190, 8057 Zürich

29. April 1992

Prof. Dr. H.-J. Güntherodt

Institut für Physik, Universität Basel, Basel

'Rastertunnelmikroskopie und verwandte Techniken - Abbilden, Spektroskopie, Modifikation und Manipulation auf atomarem und molekularem Niveau'

6. Mai 1992

Prof. Dr. H. Kroto

The School of Chemistry and Molecular Science, University of Sussex, Brighton, UK

' $\mathrm{C}_{60}$, Buckminsterfullerene, The Celestial Sphere that Fell to Earth'

13. Mai 1992

Prof. Dr. A. Heller

\section{Vorträge}

\section{Société Vaudoise des Sciences Naturelles}

Mercredi à $17.15 \mathrm{~h}$

Auditoire $\mathrm{C}$, Collège Propédeutique

Université de Lausanne, Dorigny

22 avril 1992

Prof. Dr. J.A. Robinson

Université de Zürich

'Probing the Relationships between Peptide Sequence, Conformation and Function at Receptors in the Immune System'

29 avril 1992

\section{Prof Dr. E. Lindner}

Université de Tübingen

"Catalytic and Stoichiometric Syntheses with Metal-Containing Heterocycles as Reactive Intermediates'
College of Engineering, The University of Texas, Austin, USA

'Molecular Wiring Networks for Electrical Connection of Redox Centers of Enzymes to Electrodes'

20. Mai 1992

Prof. Dr. S.E. Friberg

Department of Chemistry, Clarkson University, Potsdam, New York, USA

'The Structural Organization and Function of the Stratum Corneum Lipids - A Colloid Chemistry Problem'

\section{Laboratorium für Organische Chemie der ETH-Zürich}

Organisch-chemische Kolloquien

Montag, $16.30 \mathrm{Uhr}$

Hörsaal CHN A 31

27. April 1992

fällt aus wegen Sechseläuten

4. Mai 1992

\section{Prof. Dr. G. Folkers}

ETH, Departement Pharmazie, Zürich

'Active Site Directed Modelling of Viral Kinase Nucleoside Interaction Complexes'

11. Mai 1992

fällt aus wegen Abteilungskonferenz

18. Mai 1992

Prof. Dr. D.S. Kemp

Massachusetts Institute of Technology, Department of Chemistry, Cambridge, MA/USA

'Conformational Templates as Nucleation Sites and Reporter Functions for Formation of Polypeptide Helices and Sheets in Solution'

Prof. Dr. K. Müllen

Max-Planck-Institut für Polymerforschung, Mainz/ BRD

'Konjugierte Oligomere - je länger, je lieber?'

\section{Basler Chemische Gesellschaft}

Institut für Organische Chemie

Kleiner Hörsaal

$16.45 \mathrm{Uhr}$ 


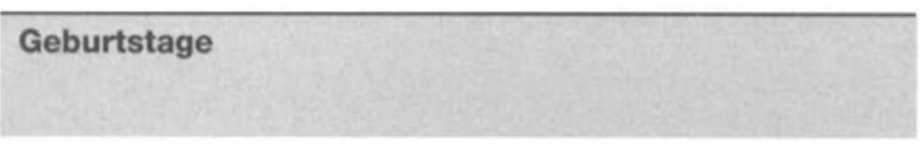

Max Rutishauser: 80. Geburtstag

Am 25. April 1992 kann Herr Dr. Max Rutishauser seinen 80. Geburtstag feiern. Anlässlich der GV vom 19.2.82 wurde er "in dankbarer Würdigung seines zielbewussten und richtungsweisenden Einsatzes als Präsident des Verbandes in den Jahren 1974 bis 1982' vom Schwei-

zerischen Chemiker-Verband zum Ehrenpräsidenten ernannt. Ein ausführliches Portrait des Jubilars erschien in Chimia 1982, 36, 176, anlässlich seines 70. Geburtstages. Der Vorstand des SChV sowie die Redaktion der Chimia entbieten die besten Glückwünsche.

\section{Akademische Ehrungen}

Prof. Dr. Albert Eschenmoser, Professor der ETH-Zürich für allgemeine organische Chemie, wurde kürzlich die Ehrendoktorwürde der Université Louis Pasteur in Strasbourg verliehen.

Prof. Dr. Dieter Seebach, Professor der ETH-Zürich für Chemie, wurde von der American Chemical Society der 'Award for Creative Work in

Synthetic Organic Chemistry' verliehen.

Prof. Dr. Wilhelm Simon, Professor derETH-Zürich für analytischeChemie, wurde die 'Talanta Medal' (Great Britain) verliehen. Unter den 13 Trägern der Talanta Medaille befinden sich auch die ehemaligen ETH-Professoren G. Schwarzenbach und $J$. Ruzicka.
7. May 1992

Prof. J. Rebek, Jr.

Massachusetts Institute of Technology, Cambridge,

USA

'Recognition and Replication in Model Systems'

\section{Chemische Gesellschaft Zürich}

Mittwoch 17.15 Uhr

Hörsaal 15-G-19 der Universität Zürich-Irchel

Winterthurerstrasse 190, 8057 Zürich

29. April 1992

Prof. Dr. H.-J. Güntherodt

Institut für Physik, Universität Basel, Basel

'Rastertunnelmikroskopie und verwandte Techniken - Abbilden, Spektroskopie, Modifikation und Manipulation auf atomarem und molekularem Niveau'

6. Mai 1992

Prof. Dr. H. Kroto

The School of Chemistry and Molecular Science, University of Sussex, Brighton, UK

' $\mathrm{C}_{60}$, Buckminsterfullerene, The Celestial Sphere that Fell to Earth'

13. Mai 1992

Prof. Dr. A. Heller

\section{Vorträge}

\section{Société Vaudoise des Sciences Naturelles}

Mercredi à $17.15 \mathrm{~h}$

Auditoire $\mathrm{C}$, Collège Propédeutique

Université de Lausanne, Dorigny

22 avril 1992

Prof. Dr. J.A. Robinson

Université de Zürich

'Probing the Relationships between Peptide Sequence, Conformation and Function at Receptors in the Immune System'

29 avril 1992

\section{Prof Dr. E. Lindner}

Université de Tübingen

"Catalytic and Stoichiometric Syntheses with Metal-Containing Heterocycles as Reactive Intermediates'
College of Engineering, The University of Texas, Austin, USA

'Molecular Wiring Networks for Electrical Connection of Redox Centers of Enzymes to Electrodes'

20. Mai 1992

Prof. Dr. S.E. Friberg

Department of Chemistry, Clarkson University, Potsdam, New York, USA

'The Structural Organization and Function of the Stratum Corneum Lipids - A Colloid Chemistry Problem'

\section{Laboratorium für Organische Chemie der ETH-Zürich}

Organisch-chemische Kolloquien

Montag, $16.30 \mathrm{Uhr}$

Hörsaal CHN A 31

27. April 1992

fällt aus wegen Sechseläuten

4. Mai 1992

\section{Prof. Dr. G. Folkers}

ETH, Departement Pharmazie, Zürich

'Active Site Directed Modelling of Viral Kinase Nucleoside Interaction Complexes'

11. Mai 1992

fällt aus wegen Abteilungskonferenz

18. Mai 1992

Prof. Dr. D.S. Kemp

Massachusetts Institute of Technology, Department of Chemistry, Cambridge, MA/USA

'Conformational Templates as Nucleation Sites and Reporter Functions for Formation of Polypeptide Helices and Sheets in Solution'

Prof. Dr. K. Müllen

Max-Planck-Institut für Polymerforschung, Mainz/ BRD

'Konjugierte Oligomere - je länger, je lieber?'

\section{Basler Chemische Gesellschaft}

Institut für Organische Chemie

Kleiner Hörsaal

$16.45 \mathrm{Uhr}$ 